\title{
Trailtements optiques ou la très grande valeur ajoutée de quelques couches nanométriqques
}

\author{
Michel LEQUIME \\ Professeur à Centrale Marseille \\ Animateur du Club Couches Minces Optiques de la SFO \\ Institut Fresnel, Équipe Couches Minces Optiques \\ michel.lequime@fresnel.fr
}

Les couches minces optiques, par leurs apports fonctionnels, présentent un intérêt important pour l'optique de haute précision. Des réalisations récentes, tant industrielles qu'académiques, illustrent l'intrication de ces deux domaines.

es traitements optiques de surfaces Lconstituent à l'évidence un élément clé de la performance des systèmes optiques dans lesquels ils interviennent. Et ce, à au moins deux titres:

- ils savent d'une part rendre efficaces des composants aussi élémentaires que des lentilles ou des miroirs, en modifiant le comportement des surfaces optiques quantifié par les coefficients de Fresnel : c'est le cas des traitements antireflets, sans lesquels la transmission d'un objectif complexe, notamment infrarouge, ne dépasserait pas quelques pourcents, ou encore celui des traitements réfléchissants, qui permettent d'exalter la luminosité d'un dispositif catoptrique ou la sélectivité spectrale d'une cavité Fabry-Perot ;

- et d'autre part, ils sont également capables de remplir des fonctions système de plus en plus élaborées, allant de la sélection d'une bande étroite de longueurs d'onde à la réjection d'un large spectre, en passant par la séparation des états de polarisation de la lumière ou encore l'égalisation spectrale de fonctions de gain intervenant dans les télécommunications optiques.

\section{Fonctions antireflets}

Comme souligné plus haut, les traitements de type antireflets sont souvent incontournables dans le domaine infrarouge, ne serait-ce qu'à cause des indices élevés des substrats (silicium, sulfure de zinc, sulfure de sélénium, germanium...) qui sont utilisables dans cette plage spectrale. On rappelle que celle-ci s'étend typiquement sur plus de 4 octaves, de 1,5 $\mu$ mà $15 \mu \mathrm{m}$ et se décompose en 3 sous-bandes, respectivement désignées par les acronymes anglais SWIR (short-wavelength infrared), MWIR (mid-wavelength infrared), et LWIR (long-wavelength infrared). Mais la distribution de Boltzmann et la loi de rayonnement du corps noir imposent aussi, pour de telles longueurs d'onde, un refroidissement des détecteurs quantiques et des systèmes de prise de vues, de sorte que ce caractère large bande s'accompagne la plupart du temps d'une exigence de compatibilité avec un environnement cryogénique.

C'est ce qui a conduit Thalès SESO à développer, dans le cadre d'un projet spatial, des traitements antireflets qui soient à la fois de très hautes performances, de très large bande, résistants aux sollicitations environnementales (chaleur humide, cyclages thermiques) et adaptés à une température d'utilisation de $80 \mathrm{~K}$. À titre d'exemple, la figure 1 présente le résultat obtenu dans le cas d'un traitement antireflet déposé sur un substrat de germanium et qui permet d'abaisser le coefficient de réflexion d'une interface d'une valeur initiale de $36 \%$ à une valeur finale n'excédant pas $0,7 \%$ en moyenne sur l'ensemble des deux bandes MWIR/LWIR.

Le repérage et la détection de cibles camouflées, qui peuvent eux aussi faire

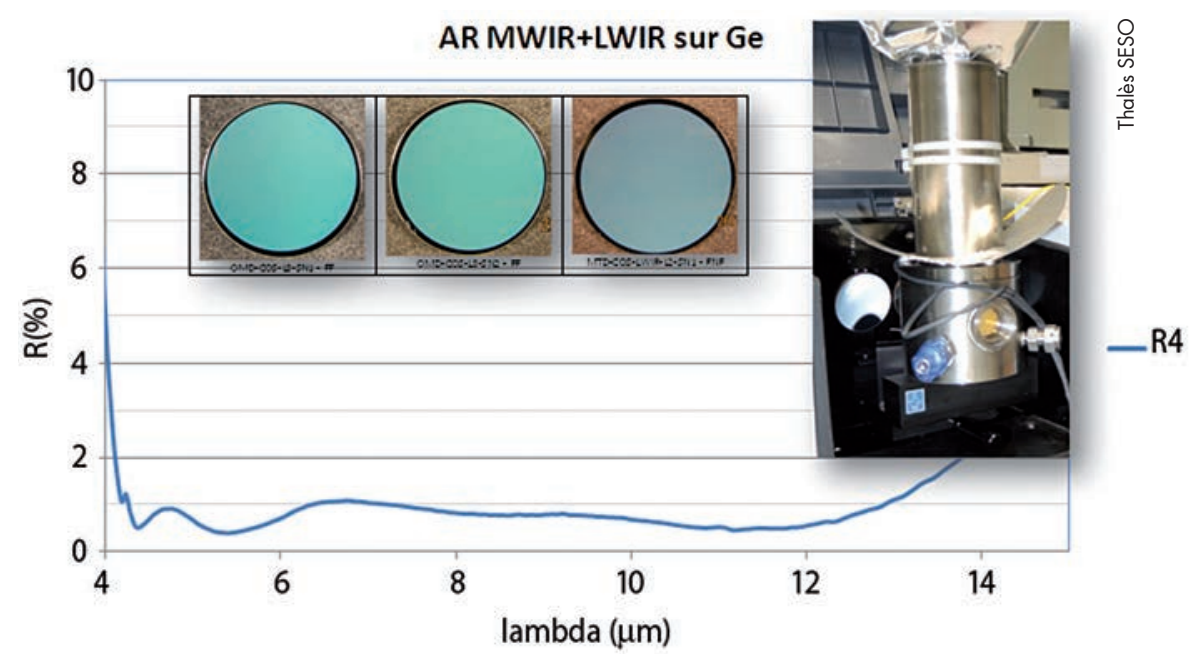

Figure 1. Dépendance spectrale du coefficient de réflexion d'une surface de germanium traitée antireflets sur l'ensemble des bandes MWIR et LWIR (mesure effectuée sous vide et à $80 \mathrm{~K}$ ). 


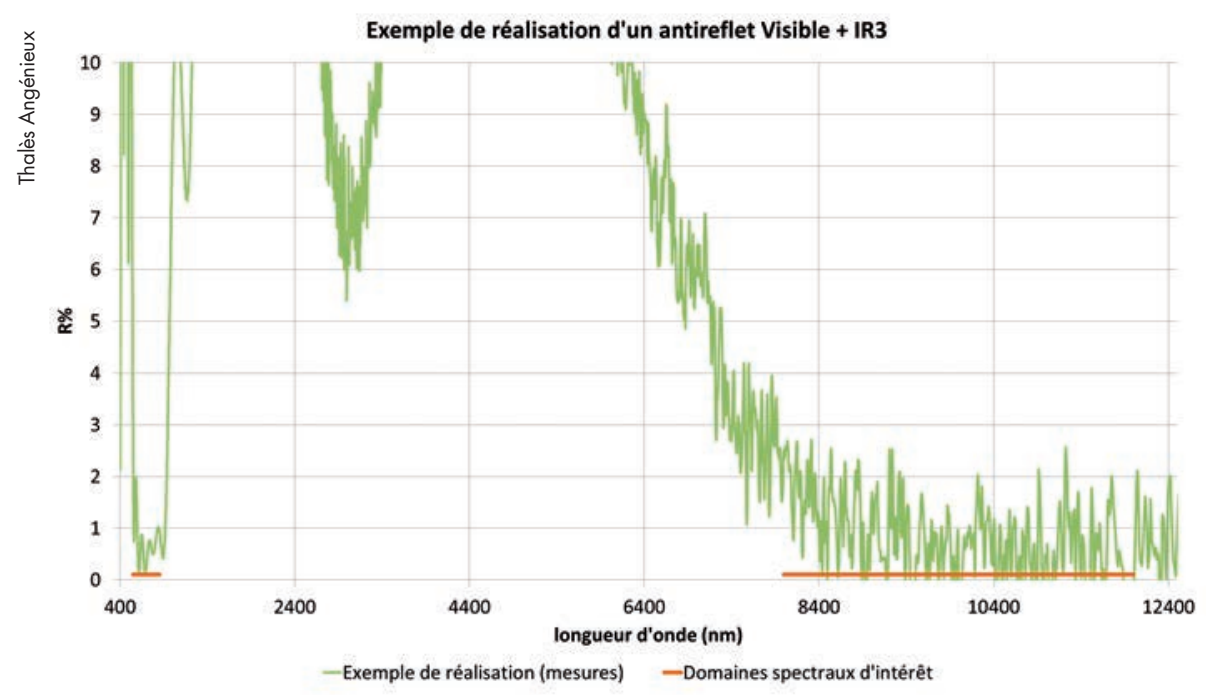

Figure 2. Performances en réflexion d'un traitement antireflets bi-bande (visible et LWIR) déposé sur un verre spécial développé par le laboratoire Verres \& Céramiques de Rennes.

appel à l'imagerie infrarouge, sont toutefois facilités par l'utilisation de systèmes de prise de vues bi-bande, c'est-à-dire susceptibles de fournir également des images dans le spectre visible, en mode BNL (bas niveau de lumière). De telles optiques multispectrales permettent en outre de simplifier les équipements en n'utilisant qu'une seule entrée pour ces 2 voies, évitant ainsi les problèmes de parallaxe et réduisant l'encombrement global du système.

C'est dans ce but que Thalès Angénieux a mis au point, dans le cadre d'un projet financé par la DGA (délégation générale de l'armement), un traitement antireflet multispectral simultanément efficace dans le visible (entre 550 et $850 \mathrm{~nm}$ ) et la bande infrarouge LWIR (entre 8 et $12 \mu \mathrm{m})$. Ce traitement a été déposé sur un nouveau verre développé dans ce but par le laboratoire Verres \& Céramiques de Rennes ${ }^{1}$. La figure 2 présente le résultat des performances en réflexion obtenues sur l'ensemble du domaine spectral d'intérêt. La réalisation de dépôts de cette qualité sur une matière entièrement nouvelle et avec d'excellentes performances en termes d'adhérence ont constitué les principaux

${ }^{1}$ Ce nouveau verre a été mis au point dans le cadre d'un projet ayant pour objectif de développer des optiques moulables multi-spectrales (visible/proche infrarouge et infrarouge thermique). II s'agit d'un verre de chalcogénures, issu du système $\mathrm{Ga}_{2} \mathrm{~S}_{3}-\mathrm{GeS}_{2}-\mathrm{CsCl}$, dont la composition a été retenue pour ses performances optiques mais également pour sa stabilité chimique et thermique. challenges de ce projet, tout comme l'importance du domaine spectral de transparence qui contraint le choix des matériaux des couches et celui de leurs conditions de dépôt.

\section{Fonctions réfléchissantes}

Le miroir est certainement le composant optique le plus répandu, en particulier lorsqu'il est plan, et l'on pourrait être étonné qu'il soit, encore aujourd'hui, l'objet de développements innovants. Mais les quelques exemples qui vont suivre devraient convaincre le lecteur du caractère pour le moins dynamique de ce secteur. Premier exemple : le réseau de télescopes constituant la très grande infrastructure

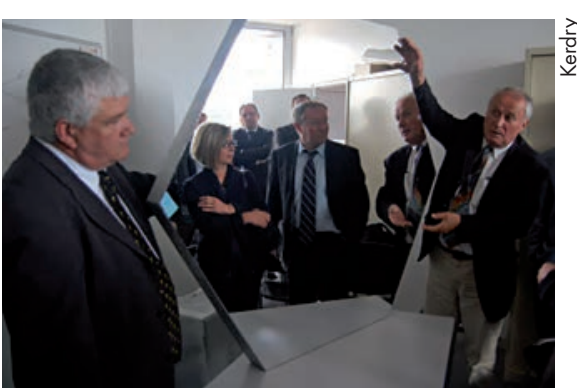

Figure 3. Prototype de miroir hexagonal traité aluminium réalisé par la société Kerdry dans le cadre du projet CTA.

de recherche désignée par l'acronyme CTA (Cherenkov Telescope Array). Celle-ci nécessite en effet la fabrication, dans sa composante MST (mid-size telescope), de miroirs de la classe 10 à 12 mètres obtenus par juxtaposition de segments hexagonaux de grandes dimensions (1,2 mètre plat à plat). La société Kerdry est impliquée dans ce projet et a su mener à bien, en collaboration avec le CEA, la fabrication et la métallisation de composants prototypes dont la structure fait massivement appel aux matériaux composites. Le traitement réfléchissant de ces pièces a nécessité de nombreuses innovations, portant aussi bien sur le procédé d'aluminisation sous vide, les procédures de nettoyage permettant d'assurer la qualité d'adhésion du traitement, ou encore la protection contre l'oxydation du dépôt une fois celui-ci réalisé. La figure 3 présente l'un de ces miroirs prototypes.

Second exemple : le Laser MegaJoule, dont on sait la place de premier plan qu'il occupe dans le paysage français de

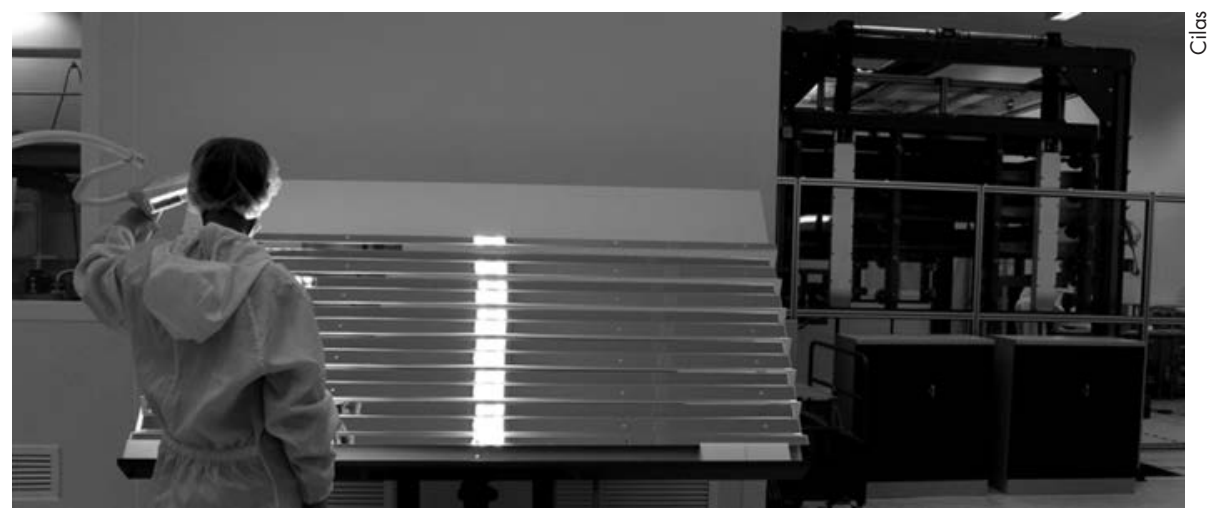

Figure 4. Réflecteur de la section amplificatrice du Laser Megaloule traité argent par la société CILAS sur sa machine PACA2M. 


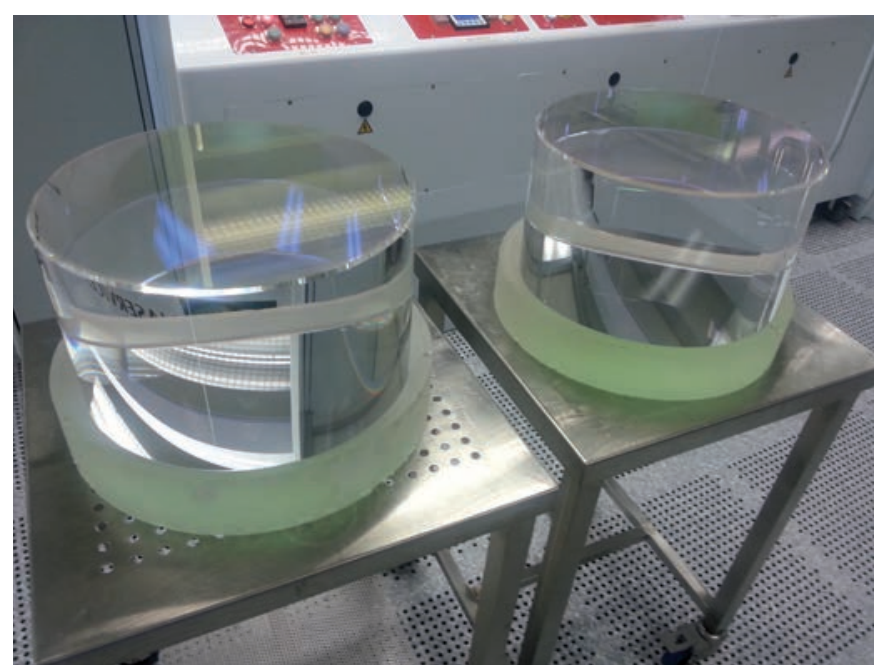

Figure 5. Miroir de fermeture de la cavité de l'interféromètre Advanced Virgo après traitement (diamètre $35 \mathrm{~cm}$, épaisseur $20 \mathrm{~cm}$ ).

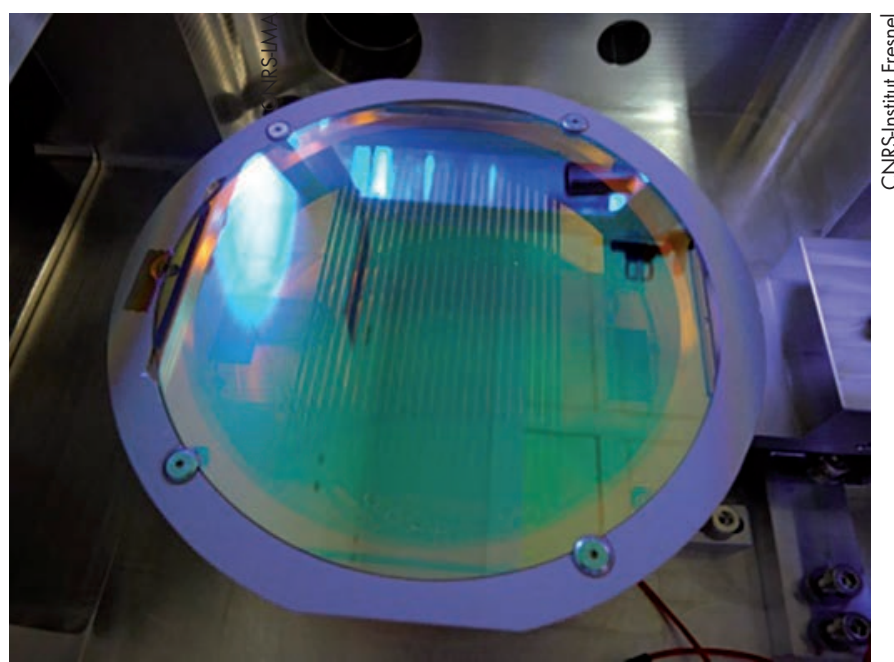

Figure 7. Wafer de filtres allumettes réalisé dans le cadre du programme CNES IDEFIX. l'optique-photonique. La société CILAS réalise ainsi, par pulvérisation magnétron, l'argenture des réflecteurs de grande taille (typiquement 2 mètres) qui équipent la section amplificatrice de ce laser de puissance développé par le CEA (figure 4). On parle ici de milliers de pièces qu'il faut traiter, mais aussi nettoyer, avant et après traitement. La technologie mise en œuvre a fait la preuve de sa qualité en termes de tenue mécanique, de reproductibilité et d'uniformité. Elle a donc pu être appliquée à la production industrielle de fonctions

de traitement variées, non seulement réfléchissantes, mais également antireflets ou absorbantes, et ce, sur un large domaine spectral allant de l'infrarouge à I'ultraviolet, grâce à la mise en œuvre d'un contrôle optique in situ adapté.

Troisième exemple : les miroirs des interféromètres gravitationnels de deuxième génération (Advanced LIGO aux USA, Advanced Virgo en Europe). Le traitement à très haute réflectivité $(99,9995 \%)$ de ces pièces de grande taille $(35 \mathrm{~cm}$ de diamètre, $20 \mathrm{~cm}$ d'épaisseur) est ici obtenu

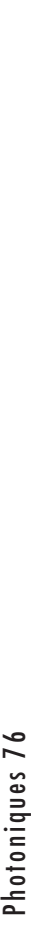

$\stackrel{2}{1}$
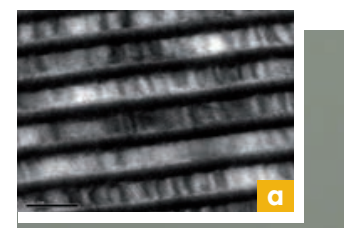
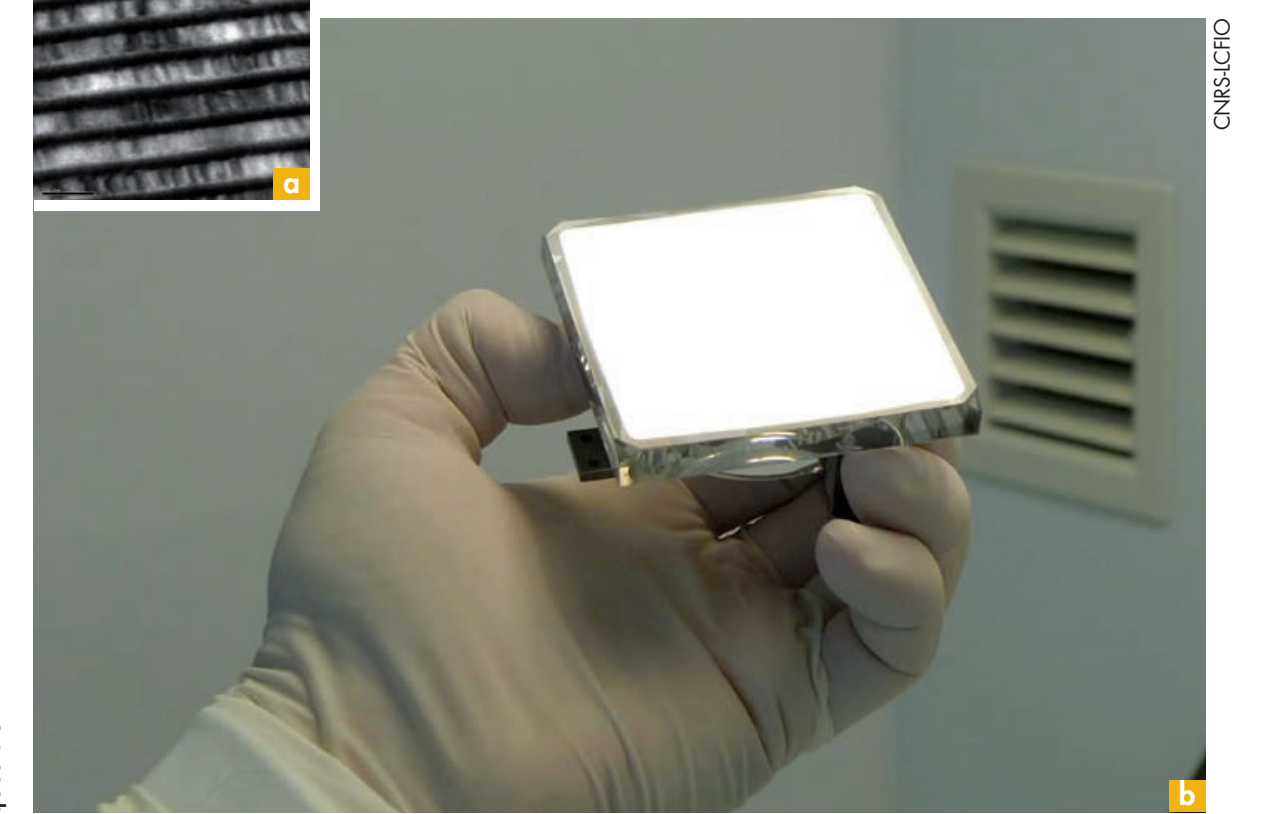

Figure 6. (a) Section transverse TEM d'un multicouche Al/Mo/SiC. (b) Miroir multicouche EUV du télescope Full Sun Imager (mission ESA Solar Orbiter). grâce au dépôt, par pulvérisation ionique, de couches alternées haut et bas indices, quart-d'onde à la longueur d'onde du laser (1064 nm). Ces dépôts ont été réalisés par le Laboratoire des Matériaux Avancés sur sa machine IBS (ion beam sputtering) dont la taille de chambre approche 2,4 m x 2,4 m $x 2,2 \mathrm{~m}$. Les quantités sont ici évidemment faibles (quelques unités), mais les performances réellement ultimes, aussi bien en termes d'absorption (0,3 ppm² à 1064 nm), de diffusion (3 ppm à 1064 nm), que d'uniformité (défaut de planéité du composant traité de 0,35 nm $\mathrm{RMS}^{3}$ sur un diamètre de $150 \mathrm{~mm}$, sachant que les substrats en silice polis par la société Zygo aux USA présentaient des planéités initiales quasi parfaites : 0,2 nm RMS sur ce même diamètre de $150 \mathrm{~mm}$ ). L'obtention d'une telle performance a nécessité la mise au point d'une technique de masquage évoluée, couplée à l'emploi d'un mouvement planétaire. La figure 5 présente deux exemplaires de ces miroirs après traitement.

Pour notre quatrième exemple, nous allons quelque peu changer d'échelle en nous translatant dans le domaine de l'extrême ultraviolet (EUV). C'est en effet dans cette plage particulière de longueurs d'onde qu'ont été réalisées de nombreuses images de la couronne solaire, notamment au

\footnotetext{
${ }^{2}$ Le «ppm» signifie «partie par million», unité utilisée par les concepteurs de couches à très faibles pertes optiques: $1 \mathrm{ppm}=10^{-6}$ ( 1 photon perdu sur 1 million) ou $10^{-4} \%$.
} ${ }^{3} \mathrm{RMS}$ (rootmean square) : valeur efficace de la grandeur. 
travers des missions SOHO (1995) et STEREO (2006). Dans le cadre de la mission ESA Solar Orbiter, dont le lancement est prévu en 2018, le laboratoire Charles Fabry de I'Institut d'Optique, en collaboration avec I'Institut d'Astrophysique Spatiale et avec le soutien du CNES, a proposé un nouveau concept de miroir multicouche permettant de réfléchir simultanément deux bandes spectrales dans I'EUV. L'introduction et la maîtrise de la croissance des couches d'aluminium dans ces empilements a permis d'améliorer de manière significative leurs performances dans cette gamme spectrale. Ainsi, un record de réflectivité de $56 \%$ à la longueur d'onde de $17 \mathrm{~nm}$ a été obtenu avec un empilement $\mathrm{Al} / \mathrm{Mo} / \mathrm{SiC}$ (pour comparaison, les revêtements multicouches Mo/Si des télescopes de STEREO réfléchissaient environ $40 \%$ à cette même longueur d'onde). La figure $6 a$ présente la section transverse de l'empilement ternaire observé au microscope électronique, tandis que la figure $6 b$ montre la taille et la forme du composant achevé.

\section{Fonction système}

Dans le cadre du projet CNES dénommé IDEFIX, l'équipe couches minces optiques de l'Institut Fresnel a développé, en partenariat avec les sociétés Cilas et Sodern, des filtres allumettes de $80 \mathrm{~mm}$ de long sur $2 \mathrm{~mm}$ de large, adaptés à l'observation multispectrale de la terre dans la gamme spectrale 300-1100 nm. La figure 7 présente une photographie d'un wafer après traitement sur lequel ont été délimitées par masquage une vingtaine d'allumettes. Ces filtres à profil spectral rectangulaire et réjection large bande ont nécessité le recours à des empilements à grand nombre de couches (plus d'une centaine par face) et forte épaisseur totale ( 25 microns et plus). Ils ont été réalisés par pulvérisation magnétron assistée, à l'aide d'une machine Leybold Optics de type Helios : les écarts entre courbes expérimentales et prédictions théoriques n'excèdent pas quelques pourcents sur l'ensemble de la gamme spectrale considérée. De plus, il a été montré que l'uniformité de la longueur d'onde de centrage était meilleure que $\pm 0,5 \%$ pour chacune de ces allumettes. Cette nouvelle technologie de dépôt ouvre donc la voie à la fabrication de fonctions de filtrage de plus en plus complexes qui ne pouvaient jusqu'à maintenant être obtenues.

\section{Conclusion}

On voit donc que l'aphorisme utilisé en titre est tout-à-fait justifié et que les traitements optiques occupent, dans tous les secteurs industriels, et donc notamment dans ceux qui ont un lien avec l'optique de précision, une place incontournable dont la valeur ajoutée ne saurait que croître dans le futur.

Remerciements à Franck Delmotte (Laboratoire Charles Fabry de I'Institut d'optique), Marie Duchêne (Thalès Angénieux), Catherine GrèzesBesset (CILAS), Jean-Claude Keromnes (Kerdry), Julien Lumeau (Institut Fresnel), Laurent Pinard (Laboratoire des Matériaux Avancés) et Patrick Robert (Thalès SESO).

\section{DS: Les capteurs CMOS, c'est l'avenir ! - Avec IDS, vous êtes sur la bonne voie!}

Sony, leader du marché mondial, va cesser sa production de capteurs CCD. Anticipant cet événement, nous avions décidé, dès 2011, d'intégrer uniquement des capteurs CMOS dans nos nouveaux modèles de caméras. Et, nous en étions déjà bien conscients, les capteurs CMOS, c'est l'avenir ! Alors, saisissez votre chance et passez aux capteurs CMOS, accompagné par un partenaire fiable et expérimenté, et ce, dans un délai de transition adapté ! Nous vous ferons profiter de plus de dix années d'expertise en matière de développement et de distribution de la technologie CMOS. Adieu les CCD!

$\mathrm{N}$ otre objectif est d'apporter aux clients la meilleure expérience utilisateur sur le marché de l'imagerie et de la vision industrielle. Forts de cette motivation, nos développeurs travaillent chaque jour avec passion pour tirer le meilleur parti de chaque capteur. En augmentant qualité d'image et performance, en créant de nouvelles fonctions tout en préservant un fonctionnement simple, afin de fournir à nos clients les meilleurs résultats dans leur application. De nouvelles perspectives dans de nouveaux champs d'application sont ouvertes.

Parmi les nombreuses fonctionnalités IDS, exceptionnelles, dont vous pouvez bénéficier, le capteur WVGA MT9V032 de Aptina permet, en fonctionnement standard, une fréquence de 60 images par seconde. IDS est le premier fabricant au monde à avoir réussi à obtenir 100 images par seconde. Soit, pour vous, près de deux fois plus d'images par seconde et, en conséquence, près du double de pièces produites, contrôlées sur un laps de temps donné. Ce qui constitue donc, pour vous, une alternative économique aux caméras rapides.

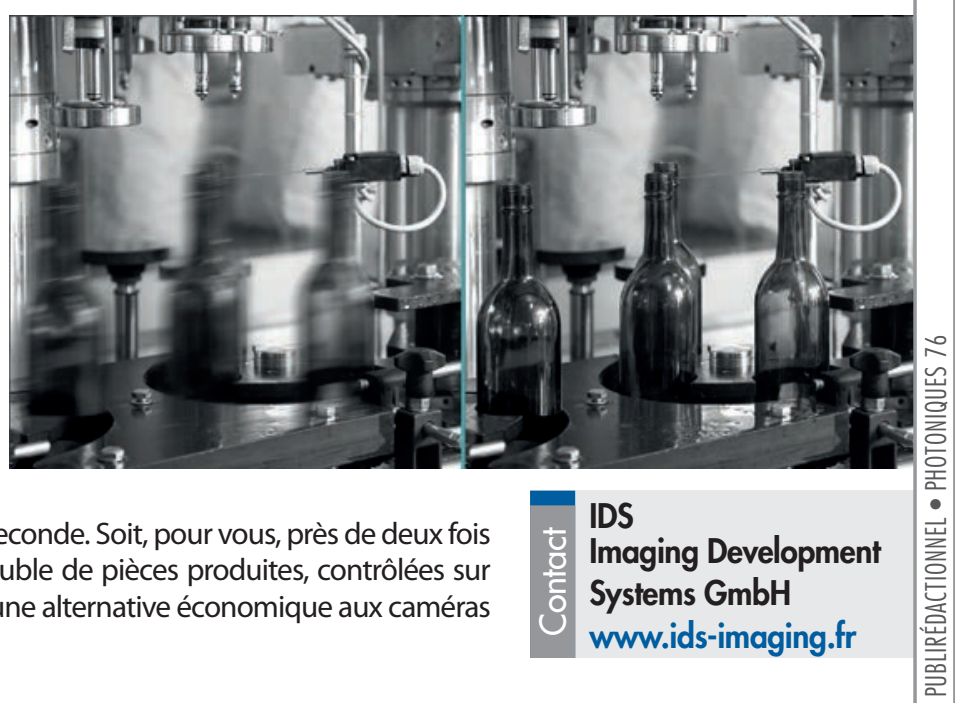

